\title{
Five new very low mass binaries ${ }^{\star}$
}

\author{
G. Montagnier ${ }^{1,2}$, D. Ségransan ${ }^{2}$, J.-L. Beuzit ${ }^{1}$, T. Forveille ${ }^{3,1}$, P. Delorme ${ }^{1}$, X. Delfosse ${ }^{1}$, C. Perrier ${ }^{1}$, S. Udry ${ }^{2}$, \\ M. Mayor ${ }^{2}$, G. Chauvin ${ }^{5}$, A.-M. Lagrange ${ }^{1}$, D. Mouillet ${ }^{4}$, T. Fusco ${ }^{6}$, P. Gigan ${ }^{7}$, and E. Stadler ${ }^{1}$ \\ ${ }^{1}$ Laboratoire d'Astrophysique de Grenoble, BP 53, 38041 Grenoble Cedex, France \\ e-mail: Guillaume.Montagnier@obs.ujf-grenoble.fr \\ 2 Observatoire de Genève, 51 chemin des Maillettes, 1290 Sauverny, Switzerland \\ 3 Canada-France-Hawaii Telescope Corporation, 65-1238 Mamalahoa Highway, Kamuela, HI 96743, Hawaii, USA \\ ${ }^{4}$ Laboratoire d'Astrophysique de Toulouse et Tarbes, BP 826, 65008 Tarbes Cedex, France \\ 5 European Southern Observatory, Casilla 19001, Santiago 19, Chile \\ 6 ONERA-DOTA, BP 72, 29 avenue de la Division Leclerc, 92322 Châtillon Cedex, France \\ 7 Laboratoire d'Études Spatiales et d'Instrumentation Astrophysique, 92195 Meudon Cedex, France
}

Received 26 July 2006 / Accepted 12 October 2006

\section{ABSTRACT}

\begin{abstract}
We report the discovery of companions to 5 nearby late M dwarfs (>M5), LHS1901, LHS4009, LHS6167, LP869-26 and WT460, and we confirm that the recently discovered mid-T brown dwarf companion to SCR1845-6357 is physically bound to that star. These discoveries result from our adaptive optics survey of all M dwarfs within $12 \mathrm{pc}$. The new companions have spectral types M5 to L1, and orbital separations between 1 and $10 \mathrm{AU}$. They add significantly to the number of late $\mathrm{M}$ dwarfs binaries in the immediate solar neighbourhood, and will improve the multiplicity statistics of late M dwarfs. The expected periods range from 3 to 130 years. Several pairs thus have good potential for accurate mass determination in this poorly sampled mass range.
\end{abstract}

Key words. binaries: visual - stars: low-mass, brown dwarfs - techniques: high angular resolution - instrumentation: adaptive optics

\section{Introduction}

Stellar masses can only be measured empirically by determining the orbits of multiple stars, and stellar multiplicity is a key parameter for several important astrophysical issues: models of stellar formation and of the subsequent dynamical evolution of multiple systems should reproduce the multiplicity fraction of every stellar class, as well as the distributions of their orbital elements, and a good handle on stellar multiplicity is crucial to correcting observed luminosity functions for unresolved components.

The stellar multiplicity statistics are now well established for solar neighbourhood G \& K dwarfs (Duquennoy \& Mayor 1991; Halbwachs et al. 2003) and is converging for M0-M5 dwarfs (Delfosse et al. 1999, and in prep.). The binary fractions clearly decrease with mass, from $57 \%$ for $\mathrm{G}$ dwarfs (Duquennoy \& Mayor 1991), to $29 \pm 5 \%$ for M0-M5 dwarfs (Delfosse et al. in prep). That fraction apparently drops further for late $\mathrm{M}$ dwarfs to $9_{-3}^{+4} \%$ beyond $3 \mathrm{AU}$ (Siegler et al. 2005), compared to $18 \pm 4 \%$ for early-M dwarfs over the same separation range. The total binary fraction of late-M dwarfs is however uncertain, because current observations have limited sensitivity to significantly closer binaries, which have been suggested to be fairly numerous (Jeffries $\&$ Maxted 2005). We are therefore observing with adaptive optics (AO) a well defined, nominally volume limited, sample of

$\star$ Based on observations obtained at the Canada-France-Hawaii Telescope (CFHT) which is operated by the National Research Council of Canada, the Institut National des Sciences de l'Univers of the Centre National de la Recherche Scientifique of France, and the University of Hawaii; and observations made at the European Southern Observatory, Paranal, Chile through proposal 075.C-0733(A) and GTO NACO. over 40 late-M dwarfs (>M5) within 12 pc (Delfosse et al., in prep.). The close limiting distance of that sample ensures optimal linear resolution, and provides good overlap with the sensitivity range of a planned search for spectroscopic binaries. In this paper we present 5 new companions to late-M primaries from that sample, and confirm that the mid-T brown dwarf companion to SCR1845-6357 (Biller et al. 2006) is physical. Section 2 describes our observations and data analysis, while Sect. 3 briefly discusses the physical parameters of the new binary systems.

\section{Observations and data reduction}

LHS 1901, LHS 4009, and LP 869-26 were observed at the Canada-France-Hawaii Telescope (CFHT), using the Adaptive Optics Bonnette (Rigaut et al. 1998) and the KIR camera, with observing procedures documented in Beuzit et al. (2004). LHS 6167, SCR 1845-6357 and WT 460 were observed with the NACO instrument (Rousset et al. 2003; Lagrange et al. 2003; Lenzen et al. 2003) on VLT UT4 (ESO Very Large Telescope, Paranal Chile). We used the IR wavefront sensor of NAOS, which produces the best corrected images for such faint red stars. The NACO observation sequences consisted of several exposures at 7 random positions within a $5^{\prime \prime}$ jitter box. The total integration time was kept $>5 \mathrm{~min}$, to average the residual speckle noise and increase the signal to noise ratio of the images. The faintest companion, SCR 1845-6357B $(\Delta(K)=5.1)$, is however easily visible in the individual raw images, illustrating the impressive performances of NACO in its classical imaging mode.

The images were reduced with the ECLIPSE package (Devillard 1997), following standard steps for near-IR 

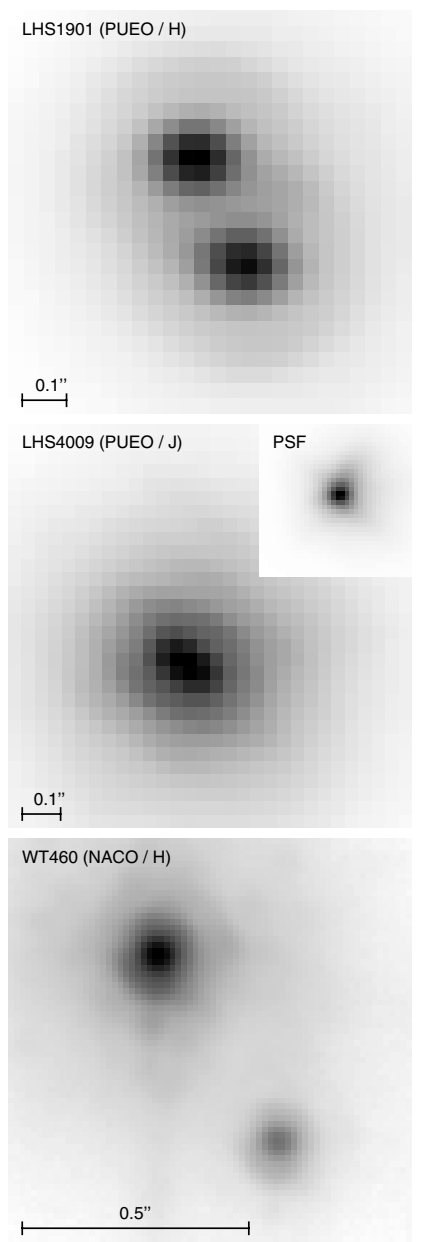

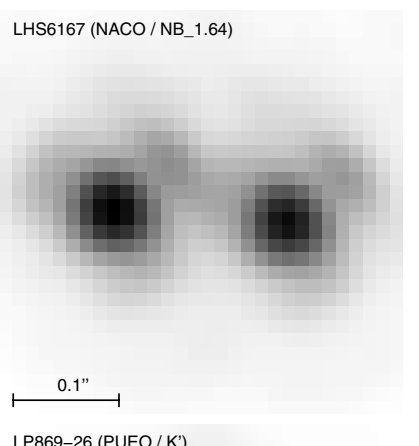

LP869-26 (PUEO / K')

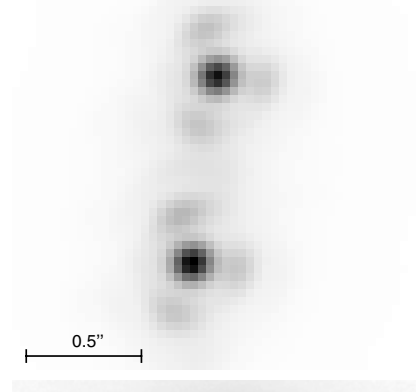

SCR1845 (NACO / H)

Fig. 1. AO images of the six resolved binaries. The images of LHS 1901, LHS 4009, LP 869-26, LHS 6167 are displayed with a linear stretch. Those of SCR 1845-6357 and WT 460 use a logarithmic stretch. North is up and East is to the left.

imaging: masking of bright and dead pixels, flat-fielding, subraction of the sky background as estimated from a median of the jittered images (iteratively masked for astronomical sources), and stacking after the images had been aligned through crosscorrelation. The flat-field map was derived from images of the illuminated dome at CFHT, and from images of the sunset sky for the VLT observations. We reconstructed the long-exposure PSF associated with each CFHT image from the data of the wavefront sensor and the deformable miror commands (Véran et al. 1997). For the NACO observation of SCR 1845-6357 we use the primary star as the reference PSF, since the two components are well separated. For the other NACO targets we make sure that the PSF is a single star observed within a few hours of the target, with the same instrumental setup and with approximately the same atmospheric conditions and resulting Strehl ratio. We extracted the coordinates and intensities of the two stars by least-squares fitting two scaled PSFs to each image (Véran et al. 1999). Astrometric calibrations derived from observations of the standard Orion field (McCaughrean \& Stauffer 1994) and of wide Hipparcos binaries then provided the position angle and separation. Figure 1 displays one reduced image for each target, and Table 2 summarizes the extracted parameters.

We computed magnitudes for the individual components from the 2MASS photometry of the systems (Skrutskie et al. 2006) and our adaptive optics flux ratio. For SCR1845-6357 we
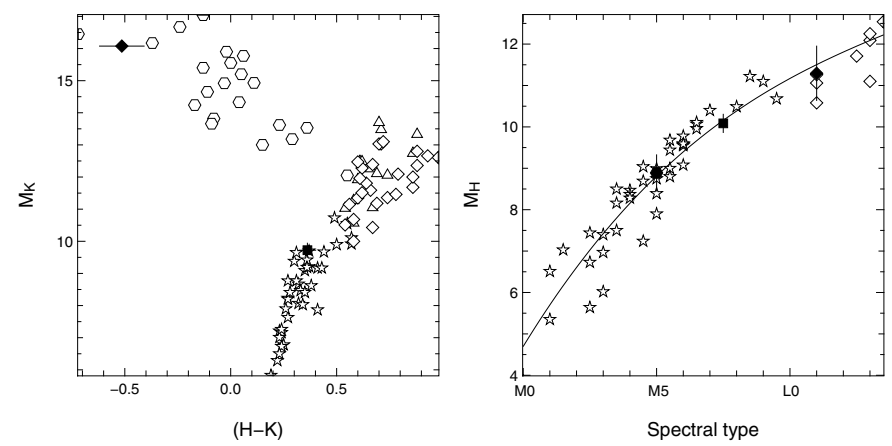

Fig. 2. Left: absolute $K$ magnitude vs $H-K$. Only secondaries observed in both $H$ and $K$ band are plotted here. The full diamond is SCR1845-6357B and the full square is LHS1901B. Right: absolute $H$ magnitude vs. spectral type. The plain line is a polynomial fit of the reference observational data points for the function $M_{H}$ (S. Type). Only secondaries observed in $H$ band are plotted here. The full hexagon is LHS6167B, the full square is LHS1901B and the full diamond is WT460B. For both figures: empty stars are M-dwarfs from Leggett et al. (2000) and Leggett et al. (2002), empty triangles are L-dwarfs from Leggett et al. (2002), empty diamonds are L-dwarfs from Knapp et al. (2004), and hexagons are T-dwarfs from Knapp et al. (2004).

used synthetic photometry from appropriate spectra from S.K. Leggett's on-line spectral database to correct the NB_1.64 flux ratio to the broader $K$ band. $\left(K-N B \_2.17=0.23 \pm 0.07\right.$ for a M8.5-Mid-T system). We then approximately corrected the photometric distances of the systems (except SCR1845-6357, which has an accurate trigonometric parallax) for the light of the previously unrecognized components, and we estimated indicative spectral types from plots of absolute magnitude versus spectral type and colour versus spectral type, derived from Leggett et al. (2000, 2002) and Knapp et al. (2004). Figure 2 shows one of these diagrams. We derived masses using the observational mass-luminosity $(M-L)$ relations of Delfosse et al. (2000) up to spectral type M7, the DUSTY model of Chabrier et al. (2000) at 1 to 5 billion years for spectral types M8 to L, and the COND models of Baraffe et al. (2003) for the mid-T SCR1845B. The estimated semi-major axes are 1.35 times the observed separation, a statistical factor between the semi-major axis of a visual binary and its projected separation at discovery from Duquennoy \& Mayor (1991). We computed approximate orbital periods from the mass and semi-major axis. They are uncertain by a factor of $\sim 3$, dominated by the (factor of $\sim 2$ ) semi-major axis uncertainty (Table 1).

\section{Discussion}

In spite of figuring in the Luyten Half Second catalogue (Luyten 1979), LHS 1901 was only recently identified as a nearby star by Reid et al. (2003), who derived a spectral type of M6.5 and a photometric distance of $8.0 \pm 0.8 \mathrm{pc}$ (assuming a single star). The new companion is clearly bound to LHS 1901: that star has a $0.73^{\prime \prime} /$ yr proper motion (Salim \& Gould 2003), and the separation changes by only $\sim 0.1^{\prime \prime}$ over $1.5 \mathrm{yr}$. Correcting the Reid et al. (2003) photometric distance for the light of the new companion moves the system out to $11.1 \mathrm{pc}$. At that distance, the absolute magnitude of the new companion corresponds to an M7.5 star, and the Delfosse et al. (2000) $K$-band $M-L$ relation predicts masses of $0.085 M_{\odot}$ and $0.082 M_{\odot}$. The expected period of approximately 20 years is consistent with the $\sim 0.1^{\prime \prime}$ observed motion over 1.5 year, and gives good prospects for accurate mass measurements within a realistic time. 
Table 1. Systems with new low-mass companions.

\begin{tabular}{ccccccccc}
\hline \hline Name & $\begin{array}{c}m_{K} \\
a\end{array}$ & $\begin{array}{c}\text { Distance } \\
(\mathrm{pc})\end{array}$ & $\begin{array}{c}\text { Spectral } \\
\text { type A }\end{array}$ & $\begin{array}{c}\text { Spectral } \\
\text { type B }\end{array}$ & $\begin{array}{c}\text { Mass A } \\
\left(M_{\odot}\right)\end{array}$ & $\begin{array}{c}\text { Mass B } \\
\left(M_{\odot}\right)\end{array}$ & $\begin{array}{c}\text { Period } \\
(\mathrm{yr})\end{array}$ & $\begin{array}{c}\text { semi-major axis } \\
(\mathrm{AU})\end{array}$ \\
\hline LHS1901 & 9.12 & $11.0 \pm 1.1$ & M6.5 & M7.5 & $0.085 \pm 0.02$ & $0.082 \pm 0.02$ & 20 & 4 \\
LHS4009 & 8.31 & $14.6 \pm 4.4$ & M5 & M5 & $0.165 \pm 0.05$ & $0.155 \pm 0.05$ & 3 & 1.3 \\
LHS6167 & 7.73 & $10.0 \pm 2.0$ & M5 & M5 & $0.14 \pm 0.05$ & $0.13 \pm 0.05$ & 6 & 2 \\
LP869-26 & 8.26 & $13.1 \pm 2.6$ & M5.5 & M6.0 & $0.15 \pm 0.05$ & $0.14 \pm 0.05$ & 130 & 15 \\
WT460 & 8.62 & $11.6 \pm 3.5$ & M6 & L1 & $0.12 \pm 0.04$ & $0.070-0.080$ & 40 & 6 \\
SCR1845 & 8.51 & $3.85 \pm 0.02^{b}$ & M8.5 & mid-T & $0.075-0.080$ & $8-65 M_{\text {Jup }}$ & 35 & 6 \\
\hline
\end{tabular}

${ }^{a} m_{K}$ from the 2MASS catalogue (Skrutskie et al. 2006); ${ }^{b}$ Henry et al., in prep.

Table 2. AO measurements of the new low-mass companions.

\begin{tabular}{|c|c|c|c|c|c|c|c|c|}
\hline Name & $\begin{array}{c}\rho \\
\left({ }^{\prime \prime}\right)\end{array}$ & $\begin{array}{c}\theta \\
\left({ }^{\circ}\right)\end{array}$ & $\Delta m$ & Date & Filt. & Intrument & $\begin{array}{c}\text { Strehl } \\
(\%)\end{array}$ & $\begin{array}{c}\text { reference } \\
\text { star }\end{array}$ \\
\hline \multirow[t]{3}{*}{ LHS1901 } & $0.275 \pm 0.005$ & $208.0 \pm 0.5$ & $0.13 \pm 0.03$ & 08 Jan. 2004 & $\bar{K}^{\prime}$ & PUEO & 37 & LP205-49 \\
\hline & $0.204 \pm 0.005$ & $215.0 \pm 0.5$ & $0.07 \pm 0.03$ & 27 Apr. 2005 & $K^{\prime}$ & PUEO & 55 & LP423-31 \\
\hline & $0.174 \pm 0.005$ & $219.6 \pm 0.5$ & $0.14 \pm 0.05$ & 14 Oct. 2005 & $H$ & PUEO & 36 & LP205-49 \\
\hline \multirow{2}{*}{ LHS4009 } & $0.068 \pm 0.007$ & $238 \pm 3$ & $0.15 \pm 0.1$ & 14 Oct. 2005 & $J$ & PUEO & 12 & HIP482 \\
\hline & $0.066 \pm 0.007$ & $250 \pm 10$ & $0.14 \pm 0.1$ & 14 Oct. 2005 & $K^{\prime}$ & PUEO & 47 & L707-74 \\
\hline \multirow[t]{2}{*}{ LHS6167 } & $0.076 \pm 0.001$ & $82.4 \pm 0.3$ & $0.12 \pm 0.01$ & 12 Sep. 2003 & $N B \_1.64$ & $\mathrm{NACO}$ & 45 & Gl317 \\
\hline & $0.172 \pm 0.001$ & $265.8 \pm 0.1$ & $0.13 \pm 0.01$ & 01 May 2005 & $N B \_1.64$ & $\mathrm{NACO}$ & 58 & G1680 \\
\hline \multirow[t]{2}{*}{ LP869-26 } & $0.813 \pm 0.005$ & $354.7 \pm 0.3$ & $0.08 \pm 0.01$ & 03 Jul. 2004 & $\bar{K}^{\prime}$ & PUEO & 53 & LP869-19 \\
\hline & $0.828 \pm 0.005$ & $353.1 \pm 0.1$ & $0.08 \pm 0.01$ & 14 Oct. 2005 & $K^{\prime}$ & PUEO & 25 & LP869-19 \\
\hline WT460 & $0.511 \pm 0.001$ & $212.6 \pm 0.1$ & $2.47 \pm 0.05$ & 01 May 2005 & $H$ & $\mathrm{NACO}$ & 54 & primary \\
\hline \multirow[t]{2}{*}{ SCR 1845} & $1.176 \pm 0.001$ & $170.22 \pm 0.08$ & $4.43 \pm 0.05$ & 01 May 2005 & $H$ & NACO & 42 & primary \\
\hline & $1.176 \pm 0.001$ & $170.22 \pm 0.08$ & $5.14 \pm 0.05$ & 01 May 2005 & $N B \_2.17$ & NACO & 30 & primary \\
\hline Biller et al. (2006) & $1.170 \pm 0.003$ & $170.20 \pm 0.13$ & $4.19_{-026}^{+0.31}$ & 28 May 2005 & $H$ & $\mathrm{NACO}+\mathrm{SDI}$ & & \\
\hline
\end{tabular}

Hawley et al. (1996) first added LHS 4009 to the solar neighborhood inventory, finding an M5 spectral type and a $10.6 \mathrm{pc}$ spectrophotometric distance. Correcting for the light of the companion moves the system out to $14.6 \mathrm{pc}$. The binary is marginally resolved in our $J$-band CFHT image, and just elongated in two $K$-band images at the $J$-band epoch and in August 2001. The August 2001 image is insufficiently resolved to derive quantitative parameters (and thus, is not included in Table 2), since the binary was then even closer. The $\sim 3$ yr period makes the system an excellent candidate for a very accurate mass determination.

Reid et al. (2002) derived a photometric distance for LHS 6167 and identified it as a solar neighbour. Scholz et al. (2005) derived a spectral type of M5 and a more acurate spectrophotometric distance. The relative motion of the components over $1.5 \mathrm{yr}$ is only $0.25^{\prime \prime}$, consistent with the $\sim 6 \mathrm{yr}$ orbital period, and excluding a background object given the $0.440^{\prime \prime} / \mathrm{yr}$ proper motion (Salim \& Gould 2003). The system has excellent potential for accurate masses.

LP 869-26 (also NLTT 48178) figures in the New Luyten 2Tenths Catalogue, and was recognized as a close neighbour by Reid et al. (2003). Scholz et al. (2005) determined an M5 spectral type, and a more accurate spectrophotometric distance. The $0.349^{\prime \prime} /$ yr proper motion of LP 869-26 (Salim \& Gould 2003) and the $1.25 \mathrm{yr}$ interval between our two observations ensure that the pair is gravitationally bound. Correcting for the light of the new companion moves the system out to $13 \mathrm{pc}$.

The WT 460 high proper motion star was discovered by Wroblewski \& Torres (1991), and identified as a member of the immediate solar neighbourhood by Patterson et al. (1998), who used VRI photometry to derive an 11 pc photometric distance. That photometry corresponds to an $\sim$ M6 spectral type. Our adaptive optics image shows a faint companion, with a magnitude which at that distance corresponds to an early-L dwarf. Since we observed WT460 at a single epoch, we must rely on external input to demonstrate that the pair is bound. The high proper motion of WT $460\left(0.77^{\prime \prime} / \mathrm{yr}\right.$, Wroblewski \& Torres 1991) ensures that it does not interfere with examination of its May 2005 position $\left(14^{\mathrm{h}} 12^{\mathrm{m}} 59^{\mathrm{s}} .56-41^{\circ} 32^{\prime} 20^{\prime} \cdot 9\right)$ in the DSS images. That position is a blank field in the Blue (May 1st 1979), Red (June 23rd 1995), and I (June 17th 1994) images, with approximate limits of $B \sim 21.5, R \sim 20.8$ and $I \sim 19.5$. This ensures that the companion either shares the proper motion of WT 460 , or is extremely red, with $B-H>10, R-H>9$ and $I-H>8$. A background star would thus have to be a T dwarf, which at $H=11.5$ would then be the second brightest known to date. The probability of finding such a rare object in the background of our small sample is vanishingly small, demonstrating that the two objects form a common proper motion pair. We also examined the April 1999 2MASS images, where WT 460 is 4.7" away from its May 2005 position. A $\Delta(H)=2.5$ background object at a $4.7^{\prime \prime}$ separation would be obvious, and the star is unresolved. WT460B is thus a bound companion, with a photometry consistent with spectral type $\sim \mathrm{L} 1$.

SCR 1845-6357 (hereafter SCR1845) is a very recent addition to the solar neighborhood inventory, discovered by Hambly et al. (2004). This M8.5 star (Henry et al. 2004) now has an accurate trigonometric parallax (Henry et al., in prep), and is the 24th closest stellar system to the Sun ${ }^{1}$. Even more recently, Biller et al. (2006) identified a very faint companion, for which they obtained a mid-T spectral type from intermediate-band photometry. We present here an independent observation of that companion, which demonstrates that systems with the observational characteristics of SCR $1845\left(\Delta H \sim 4.5\right.$ at $\left.\sim 1^{\prime \prime}\right)$ are easily accessible to standard imaging with NACO and its IR wavefront sensor, and do not require the added complication of the differential mode used by Biller et al. (2006). We use our detection to

\footnotetext{
${ }^{1}$ See: http://wWw. chara.gsu. edu/RECONS/TOP100.htm
} 
establish, if need there be, that the pair is gravitationally bound, and to derive improved broad-band photometry. Thanks to the very large proper motion of SCR1845 (2.64" / yr, Deacon et al., 2005), the one month baseline between our May 1st 2005 observation and the May 28th Biller et al. (2006) measurement is amply sufficient to establish common proper motion: while SCR 1845 moved by $0.195^{\prime \prime}$ between the two dates, the separation of the pair changed by only $0.006 \pm 0.003^{\prime \prime}$. From its position in the $M_{K}$ vs. $H-K H-R$ diagram, SCR1845B is a mid-T dwarf.

\section{References}

Baraffe, I., Chabrier, G., Barman, T. S., Allard, F., \& Hauschildt, P. H. 2003, A\&A, 402, 701

Beuzit, J.-L., Ségransan, D., Forveille, T., et al. 2004, A\&A, 425, 997

Biller, B. A., Kasper, M., Close, L. M., Brandner, W., \& Kellner, S. 2006, ApJ, 641, L141

Chabrier, G., Baraffe, I., Allard, F., \& Hauschildt, P. 2000, ApJ, 542, 464

Deacon, N., Hambly, N., Henry, T., et al. 2005, AJ, 129, 409

Delfosse, X., Forveille, T., Beuzit, J.-L., et al. 1999, A\&A, 344, 897

Delfosse, X., Forveille, T., Ségransan, D., et al. 2000, A\&A, 364, 217

Devillard, N. 1997, The Messenger, 87, 19

Duquennoy, A., \& Mayor, M. 1991, A\&A, 248, 485

Halbwachs, J. L., Mayor, M., Udry, S., \& Arenou, F. 2003, A\&A, 397, 159
Hambly, N., Henry, T., Subasavage, J., Brown, M., \& Jao, W.-C. 2004, AJ, 128, 437

Hawley, S., Gizis, J., \& Reid, I. 1996, AJ, 112, 2799

Henry, T., Subasavage, J., Brown, M., et al. 2004, AJ, 128, 2460

Jeffries, R., \& Maxted, P. 2005, Astron. Notes, 326, 944

Knapp, G. R., Leggett, S. K., Fan, X., et al. 2004, AJ, 127, 3553

Lagrange, A.-M., Chauvin, G., Fusco, T., et al. 2003, in Proc. SPIE, 4841, 860

Leggett, S., Allard, F., Dahn, C., et al. 2000, ApJ, 535, 965

Leggett, S., Golimowski, D., Fan, X., et al. 2002, ApJ, 564, 452

Lenzen, R., Hartung, M., Brandner, W., et al. 2003, in Proc. SPIE, 4841, 944

Luyten, W. J. 1979, LHS catalogue

McCaughrean, M. J., \& Stauffer, J. R. 1994, AJ, 108, 1382

Patterson, R., Ianna, P., \& Begam, M. 1998, AJ, 115, 1648

Reid, I., Cruz, K., Allen, P., et al. 2003, AJ, 126, 3007

Reid, I., Kilkenny, D., \& Cruz, K. 2002, AJ, 123, 2822

Rigaut, F., Salmon, D., Arsenault, R., et al. 1998, PASP, 110, 152

Rousset, G., Lacombe, F., Puget, P., et al. 2003, in Proc. SPIE, 4839, 140

Salim, S., \& Gould, A. 2003, ApJ, 582, 1011

Scholz, R.-D., Meusinger, H., \& Jahreiß, H. 2005, A\&A, 442, 211

Siegler, N., Close, L., Cruz, K., Martín, E., \& Reid, I. 2005, ApJ, 621, 1023

Skrutskie, M., Cutri, R., Stiening, R., et al. 2006, AJ, 131, 1163

Véran, J.-P., Rigaut, F., Maître, H., \& Rouan, D. 1997, Opt. Soc. Am. J. A, 14, 3057

Véran, J.-P., Beuzit, J.-L., \& Chaytor, D. 1999, in Astronomy with adaptive optics: present results and future programs, ed. D. Bonaccini, 691

Wroblewski, H., \& Torres, C. 1991, A\&AS, 91, 129 